\title{
Erratum: Diffraction-induced entanglement loss of orbital-angular-momentum states [Phys. Rev. A 97, 013849 (2018)]
}

\author{
Giacomo Sorelli, Vyacheslav N. Shatokhin, Filippus S. Roux, and Andreas Buchleitner
}

(Received 15 January 2019; published 30 January 2019)

DOI: 10.1103/PhysRevA.99.019904

Equation (12) for the diffracted two-photon wave function on output needs to be amended by an additional normalization factor $1 / \sqrt{1+b^{2}}$,

$$
\Psi\left(\mathbf{r}_{1}, \mathbf{r}_{2} ; L\right)=\frac{1}{\sqrt{2\left(1+b^{2}\right)}}\left[\psi_{l_{0}}^{d}\left(\mathbf{r}_{1}, L\right) \psi_{-l_{0}}^{-d}\left(\mathbf{r}_{2}, L\right)+\psi_{-l_{0}}^{d}\left(\mathbf{r}_{1}, L\right) \psi_{l_{0}}^{-d}\left(\mathbf{r}_{2}, L\right)\right],
$$

with $b$ as the overlap between the diffracted fields $\psi_{-l_{0}}^{d}(\mathbf{r})$ and $\psi_{+l_{0}}^{d}(\mathbf{r})$ as given by Eq. (17). The equations derived from Eq. (12) need to be amended accordingly

$$
\begin{aligned}
\varrho\left(\mathbf{r}_{1}, \mathbf{r}_{1}^{\prime}\right) & =\int d^{2} r_{2} \sigma\left(\mathbf{r}_{1}, \mathbf{r}_{2} ; \mathbf{r}_{1}^{\prime}, \mathbf{r}_{2}\right) \\
& =\frac{1}{2\left(1+b^{2}\right)}\left[\psi_{l_{0}}^{d}\left(\mathbf{r}_{1}\right) \psi_{l_{0}}^{d *}\left(\mathbf{r}_{1}^{\prime}\right)+b \psi_{l_{0}}^{d}\left(\mathbf{r}_{1}\right) \psi_{-l_{0}}^{d *}\left(\mathbf{r}_{1}^{\prime}\right)+b \psi_{-l_{0}}^{d}\left(\mathbf{r}_{1}\right) \psi_{l_{0}}^{d *}\left(\mathbf{r}_{1}^{\prime}\right)+\psi_{-l_{0}}^{d}\left(\mathbf{r}_{1}\right) \psi_{-l_{0}}^{d *}\left(\mathbf{r}_{1}^{\prime}\right)\right] \\
\operatorname{tr}\left(\varrho^{2}\right) & =\int d^{2} r \int d^{2} r^{\prime} \varrho\left(\mathbf{r}, \mathbf{r}^{\prime}\right) \varrho\left(\mathbf{r}^{\prime}, \mathbf{r}\right)=\frac{1+6 b^{2}+b^{4}}{2\left(1+b^{2}\right)^{2}} \\
C(|\Psi\rangle) & =\frac{1-b^{2}}{1+b^{2}}
\end{aligned}
$$

As a result, the amplitude of the concurrence minima in Figs. 4 and 5 is slightly reduced, however, without affecting the conclusion of the original paper. The corrected figures, as derived from (12)-(19) above are displayed hereafter.

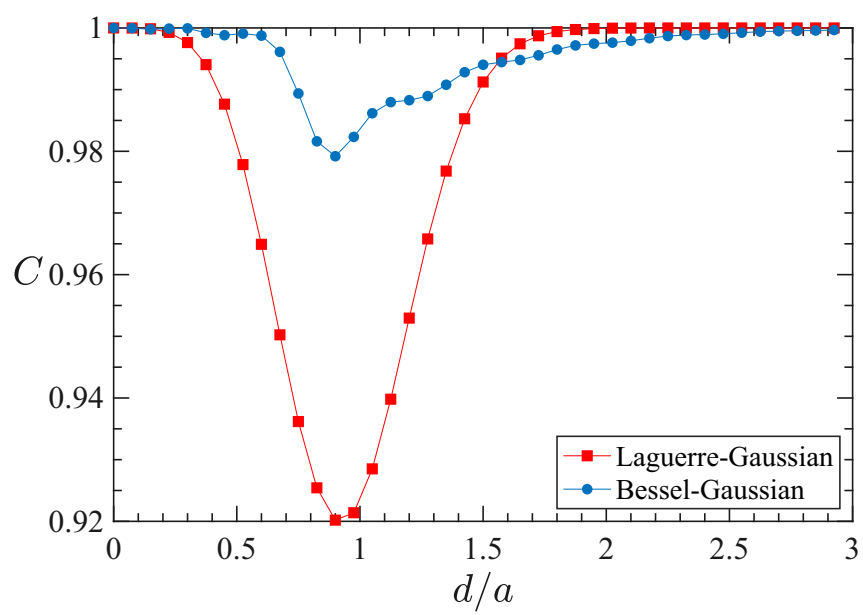

FIG. 4. Concurrence $C(|\Psi\rangle)$ vs the relative displacement $d / a(a=200 \mu \mathrm{m})$ for the diffracted twin-photon state of the Laguerre-Gaussian (LG) (red squares) and the Bessel-Gaussian (BG) (blue dots) modes with $l_{0}=1$. Beam waists were set to $w_{\mathrm{LG}}=226 \mu \mathrm{m}$ and $w_{\mathrm{BG}}=1 \mathrm{~mm}$. 

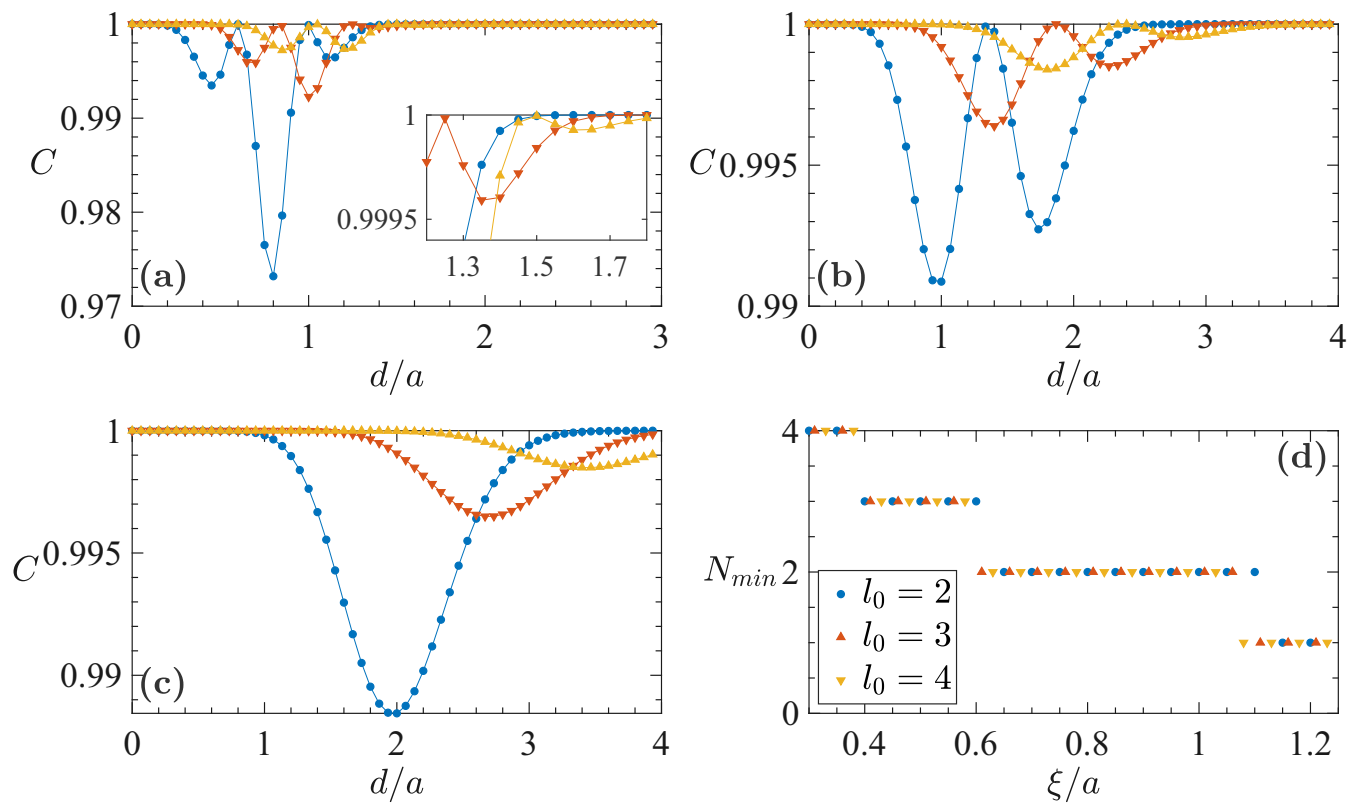

FIG. 5. (a)-(c) Concurrence $C(|\Psi\rangle)$ vs the relative displacement $d / a(a=200 \mu \mathrm{m})$ for the diffracted twin-photon state of the LG modes with $l_{0}=2-4$. Panels (a)-(c) correspond to three distinct values of the ratio $\xi / a=0.4,0.75,1.2$, respectively. (d) Number of minima of the concurrence $N_{\min }$ vs $\xi\left(l_{0}\right) / a$ for $l_{0}=2-4$. 